\title{
Syndesmosis injuries
}

\author{
Kenneth J. Hunt
}

Published online: 16 August 2013

(C) Springer Science+Business Media New York 2013

\begin{abstract}
Traumatic injuries to the distal tibiofibular syndesmosis commonly result from high-energy ankle injuries. They can occur as isolated ligamentous injuries and can be associated with ankle fractures. Syndesmotic injuries can create a diagnostic and therapeutic challenge for musculoskeletal physicians. Recent literature has added considerably to the body of knowledge pertaining to injury mechanics and treatment outcomes, but there remain a number of controversies regarding diagnostic tests, implants, techniques, and postoperative protocols. Use of the novel suture button device has increased in recent years and shows some promise in clinical and cadaveric studies. This article contains a review of syndesmosis injuries, including anatomy and biomechanics, diagnosis, classification, and treatment options.
\end{abstract}

Keywords Syndesmosis injury $\cdot$ High ankle sprain $\cdot$ Pronation external rotation $\cdot$ Sports injury $\cdot$ TightRope $\cdot$ Syndesmotic screws

\section{Introduction}

Injuries to the distal tibiofibular syndesmosis commonly result from high-energy ankle injuries and contact sports. They can occur as isolated ligamentous injuries, as seen in contact sports, or associated with ankle fractures. Syndesmotic injuries can create a diagnostic and therapeutic challenge for musculoskeletal physicians. While recent literature has added considerably to the body of knowledge pertaining to injury mechanics and treatment outcomes, there remain a number of controversies regarding diagnostic tests, implants, techniques,

\section{K. J. Hunt $(\bowtie)$}

Department of Orthopaedic Surgery, Stanford University,

450 Broadway Street, MC 6342, Redwood City, CA 94063, USA

e-mail: kjhunt@stanford.edu and postoperative protocols. Herein is a review of the current data on injuries to the tibiofibular syndesmosis.

\section{Anatomy of the syndesmosis}

The tibiofibular syndesmosis is a fibrous joint joining the fibula to the tibia and stabilized by four lateral ligaments: the anterior inferior tibiofibular ligament (AITFL), the interosseous ligament (IOL), the transverse ligament (TL), and the posterior inferior tibiofibular ligament (PITFL) (Fig. 1). At the base of the syndesmosis, there is a small contact zone where the tibia and fibula directly articulate with a small band of hyaline cartilage about $0.5-1.0 \mathrm{~mm}$ thick that is contiguous with the articular surfaces of the respective bones $[1 \bullet \bullet$. The convex fibular part of the syndesmosis is congruent with the concave incisura on the tibial side (Fig. 1). The anterior tubercle of the tibial incisura is larger than the posterior tubercle, preventing forward slipping of the distal fibula. In syndesmotic injuries that result from external rotation, the posterior tubercle functions as a fulcrum about which the distal fibula spins around its longitudinal axis in a lateral direction. A synovial-lined syndesmotic recess is usually present, bordered cranially by the distal interosseous ligament and anteriorly by the AITFL, resulting in a synovial recess within the tibiotalar joint. The width of the syndesmotic recess is $2 \mathrm{~mm}[2,3]$, and syndesmotic disruption results in injury to this membrane with increased widening, which can be easily seen arthroscopically [4]. The strong, flat, and triangularly shaped deltoid ligament on the medial side of the ankle is a key contributor to syndesmotic stability.

The blood supply to the syndesmosis has been well documented [5]. There are three distinct anterior vascular patterns from the anterior tibial and peroneal arteries and two distinct posterior vascular patterns from the peroneal and posterior tibial arteries. These patterns vary by individual and can even differ side to side within one individual. The perforating 


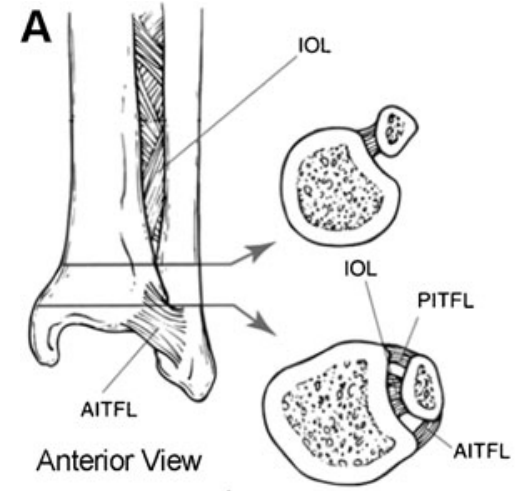

Fig. 1 Line drawings depicting anterior (a), posterior (b), and lateral (c) views of the ligaments stabilizing the distal tibiofibular syndesmosis: the anterior-inferior tibiofibular ligament (AITFL), the posterior-inferior tibiofibular ligament (PITFL), the transverse ligament (TL), and the

branch of the peroneal artery, an important blood supply to the anterior syndesmosis, is located about $3 \mathrm{~cm}$ above the joint line and is closely associated with the IOL, making it vulnerable to injury during syndesomotic disruption and surgical treatment [5].

\section{Biomechanics of the syndesmosis}

The ligaments stabilizing the syndesmosis prevent excess fibular motion in multiple directions: anterior-posterior translation, lateral translation, and internal and external rotation. Appropriate fibular position and limited rotation are necessary for normal syndesmotic function and talar position within the ankle mortise [6]. On the medial ankle, the deltoid ligament plays an important role in syndesmotic stability. Sequential disruption of the syndesmotic ligaments, as in a high-energy external rotation ankle injury (i.e., "high ankle sprain" or pronation external rotation ankle fracture) can result in excess lateral translation and rotation of the talus and fibula relative to the tibia [7].

\section{Injury mechanics}

Pathologic forces to the ankle syndesmosis typically result from excessive external rotation of the ankle at the end-range of dorsiflexion or some combination of ankle dorsiflexion associated with adduction or abduction of the foot. These forces can produce widening of the fibula relative to the tibia at the ankle mortise, disrupting the syndesmotic ligaments and resulting in secondary talar instability [8]. With high pathologic forces rotating a fixed foot (i.e., from body weight or impact with another player or object), the talus rotates laterally, resulting in injury to the AITFL. With continued force, the fibula moves further away from the tibia, producing a shearing force that can be transferred axially between the bones,

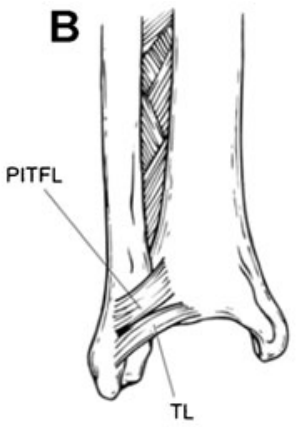

Posterior View

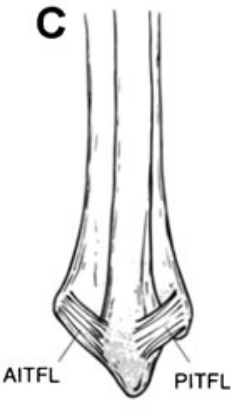

Lateral View interosseous ligament (IOL). The arrows indicate the respective location and point to the cross-sectional view. (Adapted from Hamilton CC: Traumatic Disorders of the Ankle. New York, NY: Springer-Verlag, 1984, with permission )

causing injury to the interosseous membrane [9]. With continued high forces, the PITFL and deltoid can fail, and/or the fibula, posterior malleolus, or medial malleolus can fracture.

Cadaveric studies have demonstrated that foot position impacts the nature of an injury. Haraguchi and Armiger [10] showed that external rotation of the foot while in pronation first produced damage to the AITFL, followed by medial injury to the deltoid ligament. Wei et al. [11]showed that ankle external rotation with an everted foot is also more likely to disrupt the AITFL, first producing lateral translation and external rotation of the talus. Conversely, external rotation of a neutral foot is more likely to initially result in deltoid ligament injury [11], with subsequent injury to the AITFL and syndesmotic ligaments [12]. Disruption of at least two lateral ligaments and injury to the deltoid ligament are necessary for complete syndesmosis instability. However, isolated disruption of the deltoid ligament can produce medial clear space widening even with a stable syndesmosis [13].

With progressive injury to the syndesmotic ligaments, increasing diastasis will occur between the tibia and the fibula. The normal radiographic tibiofibular clear space is approximately $5 \mathrm{~mm}$ [14]. Increased widening of the ankle mortise by as little as $1 \mathrm{~mm}$ decreases the contact area of the tibiotalar joint by $42 \%$, causing significant ankle instability[1••]. The dramatic change in ankle joint mechanics that can occur with even mild syndesmotic injuries is a likely contributor to both the prolonged recovery and long-term dysfunction associated with syndesmotic injuries $[15,16]$.

\section{Diagnosis and classification of syndesmotic injuries}

\section{Clinical diagnosis}

The diagnosis of syndesmosis injury is based on injury pattern, thorough physical examination, and radiographic findings. 
When no fracture is present, clinical findings will include ankle pain, tenderness directly over the anterior syndesmosis, and positive squeeze and external rotation tests. The squeeze test is performed with squeezing of the leg above the midpoint in the calf, producing proximal compression of the fibula and tibia above the midpoint of the calf and creating separation of the two bones distally and pain at syndesmosis [17]. It is important to note that this test can be painful proximally in the presence of a Maissoneuve injury. The external rotation test is performed by stabilizing the tibia with the knee flexed at $90^{\circ}$ and externally rotating the foot. A positive examination is noted if pain is prompted at the syndesmosis during this maneuver. Both of these tests have a high specificity but low sensitivity, with MRI scans as the gold standard [18].

\section{Radiographic diagnosis}

Radiographic evaluation should include weight bearing (where tolerated by the patient) and three views of the ankle joint (anteroposterior, mortise, lateral). Tibia/fibula x-rays should be obtained if there is clinical suspicion of fracture, particularly high in the fibula. In addition to diagnosing fracture and proximal fibula injury, radiographs can be useful in demonstrating disruption of the normal relationship between the distal tibia and distal fibula, which may be indicative of syndesmotic injury. Classically, syndesmotic injuries may be present if radiographs show increased tibiofibular clear space, decreased tibiofibular overlap, and/or increased medial clear space (Fig. 2, Table 1) [19, 20].

Evidence of syndesmotic injury is not always apparent on static injury radiographs. Beumer et al. [19] showed that there is no optimal radiographic parameter to assess syndesmotic injury. The most useful parameters are the presence of both
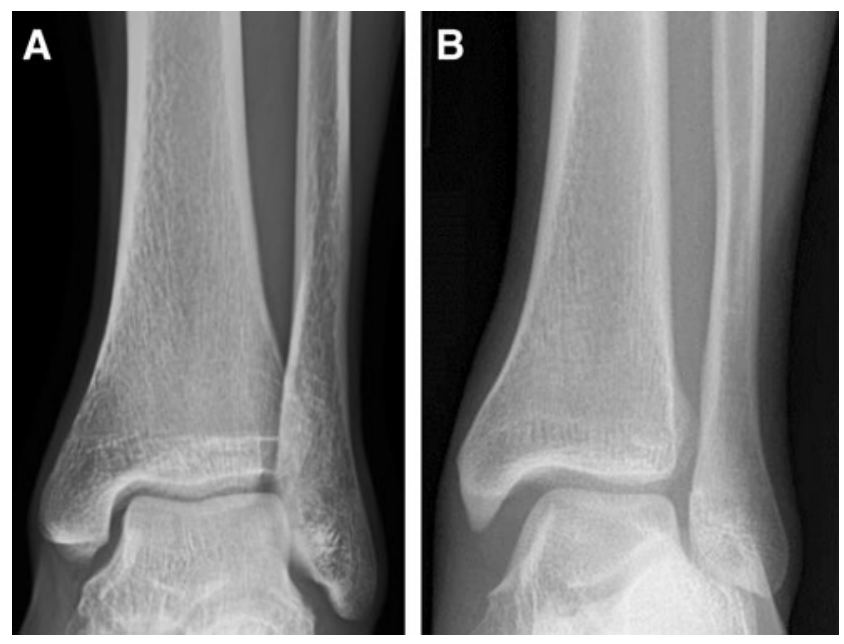

Fig. 2 Anteroposterior radiographs of a normal ankle (a) and an ankle with syndesmotic injury (b). Note the widened medial clear space, loss of tibiofibular overlap, and widening of the distal tibiofibular syndesmosis
Table 1 Radiographic parameters of syndesmotic injury

\begin{tabular}{|c|c|}
\hline $\begin{array}{l}\text { Tibiofibular clear } \\
\text { space }\end{array}$ & $\begin{array}{l}\text { - Distance between the medial border of the fibula } \\
\text { and the lateral border of the posterior aspect of the } \\
\text { tibial incisura. } \\
\text { - Should be measured } 1 \mathrm{~cm} \text { proximal to the plafond } \\
\text { - Should be less than } 6 \mathrm{~mm} \text { in both the AP and } \\
\text { mortise views }\end{array}$ \\
\hline $\begin{array}{l}\text { Tibiofibular } \\
\text { overlap }\end{array}$ & $\begin{array}{l}\text { - Overlap of the lateral malleolus and the anterior } \\
\text { tibial tubercle } \\
\text { - Measured } 1 \mathrm{~cm} \text { proximal to the plafond } \\
\text { - In the AP view, the overlap should be greater than } \\
6 \mathrm{~mm} \text { or } 42 \% \text { of the width of the fibula } \\
\text { - In the mortise view, it should be at least } 1 \mathrm{~mm} \\
\text { - Absence of tibiofibular overlap can be present as } \\
\text { an anatomic variant [22] }\end{array}$ \\
\hline $\begin{array}{l}\text { Medial clear space } \\
\text { (MCS) }\end{array}$ & $\begin{array}{l}\text { Distance between the lateral border of the medial } \\
\text { malleolus and the medial border of the talus, } \\
\text { measured at the level of the talar dome } \\
\text { - In the mortise view, MCS should be equal to or } \\
\text { less than the superior clear space between the talar } \\
\text { dome and the tibial plafond [19]. } \\
\text { - An increase in MCS indicates a deltoid ligament } \\
\text { injury. } \\
\text { - Increased tibiofibular clear space is considered the } \\
\text { most reliable indicator of syndesmotic injury [71] }\end{array}$ \\
\hline
\end{tabular}

loss of tibiofibular overlap and widening of the medial clear space, since absence of tibiofibular overlap may indicate syndesmosis widening and a medial clear space larger than a superior clear space indicates deltoid disruption [19]. Stress radiographs may be useful for diagnosing syndesmotic injury and defining indications for surgery. However, Parikenin et al. showed that intraoperative stress radiography (lateral translation and external rotation) has very high specificity but quite poor sensitivity [21]. This means that more severe injuries are fairly easily recognized, but moderate injuries with instability may be quite easily missed with stress radiography, even under anesthesia. Contralateral radiographs can also be useful. Standing x-rays can give an indication of anatomic normal for an individual patient, which can vary considerably [22], and intraoperative use of a true lateral $x$-ray can help confirm reduction in the coronal plane. Intraoperative computerized tomography (CT) scanning has been shown to be a useful tool for diagnosing syndesmotic injury and confirming syndesmosis reduction [23], but this technology is not yet widely prevalent in practice.

In the absence of an optimal plain radiographic study, MRI and CT scans can be useful static tools for assessing syndesmotic disruption, extent of ligament injury, and the position of the fibula in the syndesmosis. MRI has been shown to have high accuracy in detecting injury ( $96 \%$ ), as compared with AP x-ray (63\%) and mortise x-ray $(71 \%)[4,24]$. CT scanning is more accurate than radiographs in showing the relationship of the distal tibia and fibula [25]. CT also readily allows measurement of the contralateral ankle for comparison. A displacement difference of $2 \mathrm{~mm}$ or more side to side is 
considered pathologic. Arthroscopy is likely the best definitive tool for assessing syndesmosis injury and widening with $100 \%$ accuracy [4] but is not always feasible for diagnosis. In surgical cases, we will routinely use arthroscopy to confirm the diagnosis and reduction after stabilization [26].

\section{Classification}

Classification systems of the severity of acute syndesmosis injury have been developed by several authors [16, 27, 28]. There is general agreement that there are three grades, rated from grade I to III from least to most severe. While these classification schemes have similarities, there are also important differences between the scales. Of note, all of these scales incorporate clinical findings, but there is no current classification system that uses anatomic location or severity of ligamentous injury as defined by MRI or ultrasound, and no system provides adequate treatment guidance or prognosis.

\section{Grade I}

There is general agreement that grade I injuries are clinically mild, with a stable syndesmotic joint and normal radiographs. There is incomplete injury to the lateral ligaments. These patients will have tenderness at the syndesmosis [28]. External rotation and squeeze tests can be negative [27], although Gerber et al. indicated that one of these two tests should be positive [16].

\section{Grade II}

Grade II injuries are generally associated with complete AITFL and IOL disruption. Radiographs are normal, and external rotation and squeeze tests are positive. However, there is no consensus regarding joint stability. Scranton suggests that grade II injuries are unstable, whereas Wolf and Amendola indicate that they can be either stable or unstable. Laboratory data suggest that injury to the PITFL and transverse ligament are the key to syndesmotic stability. No current classification system helps differentiate between grade II injuries requiring stabilization and those that do not. Since the decision to stabilize surgically depends on stability, the optimal classification system would account for this and likely include MRI findings as a more accurate tool than radiographs.

\section{Grade III}

A grade III injury is a complete injury to the lateral ligaments (AITFL, IOL, PITFL) and deltoid ligament avulsion. The joint is clearly unstable with plain radiographs (greater than $2 \mathrm{~mm}$ of medial clear space widening and/or widened syndesmosis)
[29]. All clinical tests are positive. Grade III injuries require operative stabilization.

\section{Ankle fractures with syndesmotic injuries}

The large majority of data on the surgical treatment of syndesmosis injuries are in fracture-associated syndesmotic disruptions. Approximately one in seven ankle fractures are associated with an injury to the syndesmosis [12]. Rotational ankle injuries can result in particularly high mechanical forces when they occur in high-impact and collision activities, especially when they occur at high speeds and in patients with high body mass index. When forces are great enough, fractures can occur at the medial and posterior malleoli, the fibula proximal to the syndesmosis articulation, and/or the attachment sites of the syndesmotic ligaments (i.e., Chaput's and Wagstaff's tubercle) (Fig. 1). These injuries are described in the most commonly recognized fracture classifications based on fracture location (i.e., Lauge Hansen pronation external rotation injuries, supination external rotation injuries, and Danis Weber $\mathrm{C}$ injuries) (Fig. 3) [30]. It is important to note that at least $20 \%$ of syndesmotic injuries are associated with Weber B fracture patterns [31].

While the most common fracture location in syndesmotic injury is the distal third of the fibula (Fig. 3), the fracture level is related to the extent of ligamentous disruption of the
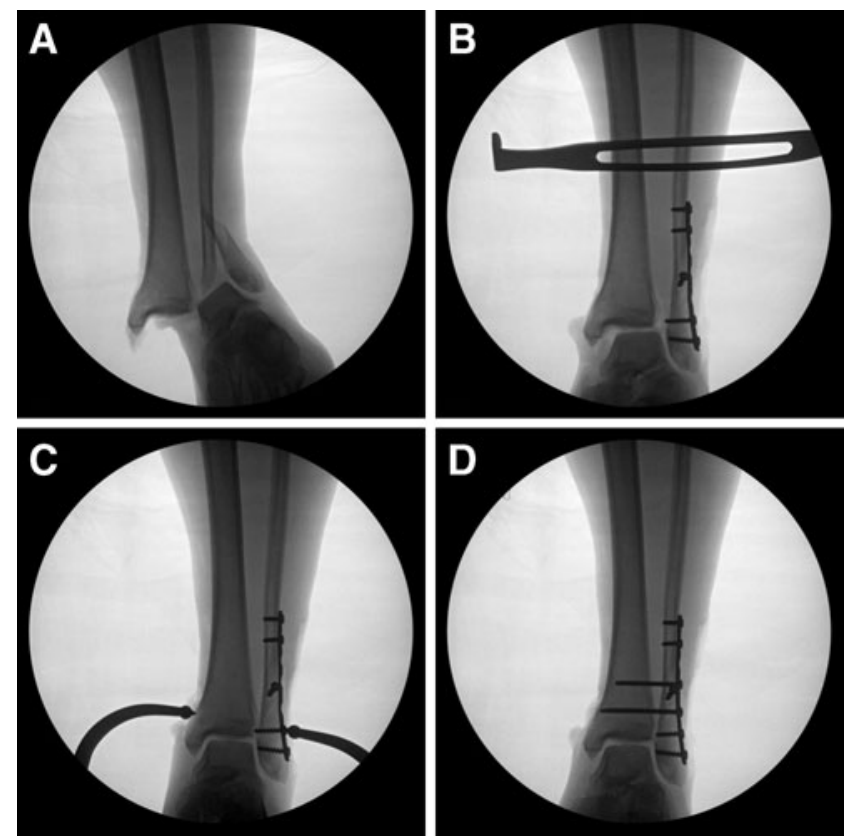

Fig. 3 Fluoroscopic images illustrating unstable supination external rotation fracture pattern with syndesmotic disruption (a), lateral translation stress after fixation of the fibula fracture (b), syndesmosis reduction technique using a large reduction tenaculum (c), and fixation of the syndesmosis with 3.5-mm screws (d) 
syndesmosis. This usually involves rupture of the AITFL and interosseous membrane, with PITFL disruption (or posterior malleolus injury) in severe injuries. Disruption of the syndesmotic ligaments can also result in a fracture at the proximal fibula, or "high fibular fracture," referred to as a Maisonneuve injury. Maisonneuve fractures generally occur with extensive rupture of the interosseous membrane [9]. These are indicative of severe trauma to the syndesmotic ligaments.

\section{Ligamentous syndesmotic injuries}

Sports activities played at high speeds, on uneven terrain, or artificial surfaces with cleated sports shoes can create or increase the likelihood of dorsiflexion and external rotation of the foot and ankle relative to the tibia $[32 \cdot, 33]$. Such injuries typically occur during impact and collision activities that involve jumping and landing maneuvers (i.e., football, soccer, basketball, rugby, skiing, hockey, etc.), which are known to result in external rotation torque, increasing the incidence of syndesmotic ankle sprain. Ligamentous injuries to the syndesmosis are commonly referred to as "high ankle sprains," since they occur proximal to the more common inversion lateral ankle sprains. These injuries can be generally classified as incomplete ligamentous injuries (i.e., "sprain") or complete ligamentous disruption. Complete ligamentous injuries are rare without fracture but are generally repaired surgically [32•]. High ankle sprains are less common than "inversion" lateral ankle sprains, comprising around $10 \%$ of all ankle sprains [15, 34], but represent up to $25 \%$ of ankle sprains in collision sports such as American football [16, 32•, 35]. As compared with inversion sprains, high ankle sprains are more likely to create long-term dysfunction [16] and require much more time for recovery $[15,36]$.

\section{Treatment of syndesmotic injuries}

\section{Conservative treatment}

Lower grade (grades I and II) isolated syndesmotic sprains can generally be successfully treated nonsurgically, since they do not result in diastasis and complete ligamentous disruption [32•]. However, these can take up to 3 times longer to heal than inversion ankle sprains. Injuries that occur in conjunction with a fracture and those with clear destabilization of the mortise generally require surgical treatment of the fracture. In these injuries, syndesmosis instability can be assessed intraoperatively in order to determine the need for syndesmosis stabilization (Fig. 3).

For appropriate injuries, conservative management of stable injuries has shown good results $[16,37]$ and generally involves the typical three-phase approach. Nassbaum et al. [37] treated 60 collegiate athletes with clinically detected "high ankle sprains" with a rehabilitation program that included short period (1-4 days) of non-weight-bearing and immobilization in a boot, followed by an aggressive rehabilitation schedule. The mean return to sport was 13.4 weeks, and time to return was statistically related to the interosseous tenderness length positive squeeze test. Since they did not obtain MRI scans, it is possible that some athletes in their series may have had minor lateral ligament sprains, which can clinically simulate syndesmotic injuries and have a tendency to recover much faster.

For higher grade injuries, surgical treatment is likely superior to nonoperative treatment, even for purely ligamentous injuries [38, 39]. Kennedy et al. [38]compared grade III syndesmotic injuries treated surgically with conservative treatment in a cast, and while there was little long-term difference in symptoms and athletic performance, return to play was, on average, 3 weeks faster in the surgical group. For grade II injuries, where there is no evidence of instability on plain radiographs or stress testing but MRI or ultrasound studies suggest a higher grade injury (i.e., complete syndesmosis ligament disruption, deltoid injury, etc.) with possible dynamic instability, arthroscopy is a useful tool for accurately assessing the injury for dynamic instability, and stabilization can be instituted at the same time as necessary [27].

\section{Surgical treatment}

Most syndesmotic injuries that occur with fractures of the fibula and/or posterior malleolus will require surgical stabilization. Isolated ligamentous injuries that result in complete syndesmotic disruption of the syndesmosis are not common, but they also require surgical stabilization to optimize shortand long-term outcomes. While a number of techniques have been described for stabilization of the syndesmosis [40-46], the most commonly used methods by far, according to recent literature, are screws and suture buttons $[6,39,41,47-50$, $51 \bullet \cdot, 52,53 \bullet, 54]$. Outcomes of both treatments are generally very good. The most important clinical predictor of outcome is consistently reported as anatomic reduction of the syndesmosis $[31,51 \bullet \bullet, 55 \bullet$. The unplanned reoperation rate for syndesmotic injuries has been reported in large series to be as high as $27 \%$ [56•]. In a study by Symeonidis et al. [56•], the most common causes of reoperation was missed syndesmotic injuries (47\%), failure to achieve anatomic reduction (31\%), and loss of reduction due to fixation failure $(21 \%)$.

Trans-syndesmotic screws

Trans-syndesmotic screws are a highly effective method for achieving stable fixation, allowing the syndesmotic ligaments 
to heal with appropriate aftercare (Fig. 3). There exists an extensive literature pertaining to the technical attributes of syndesmotic screw materials and configuration. Rates of fixation failure do not appear to be different when stainless steel screws are compared with titanium screws [57]. Larger, 4.5$\mathrm{mm}$ screws provide greater resistance to shear stress than do 3.5-mm screws [58], but cadaver testing suggests that there is no biomechanical advantage based on screw type during pronation external rotation injury [59]. Two syndesmotic screws provide superior stability, as compared with one [45], but biomechanical stability and outcomes are no different when three-cortical is compared with four-cortical screw placement $[48,57,59,60]$. The position of the foot during insertion of the syndesmotic screw does not impact final ankle range-of-motion or clinical outcome [61, 62].

\section{Complications with screw fixation}

Significant malreduction of the tibiofibular syndesmosis has been reported in up to half of patients treated with syndesmotic screws $[25,48,51 \bullet \cdot$. Malreduction has been found to be a primary predictor of clinical outcome [31, $51 \bullet \bullet, 55 \bullet, 63]$. There is some controversy regarding the necessity for screw removal too [64•]. Recent studies suggest that removal of syndesmotic screws does not impact clinical outcome. In fact, paradoxically, patients with broken screws have slightly better outcomes than do those with intact screws [64•, $65,66]$. Furthermore, a complication rate of up to $15.8 \%$ (wound infection or recurrent diastasis) has been reported after screw removal [67]. In general, screws should be left in place for at least 3 months, and removal can be justified when (1) the screw results in local tenderness or other physical complaints, (2) dorsiflexion is hindered, or (3) the patient prefers removal after informed discussion that includes concerns for potential hardware breakage or loosening. According to Schepers [64•], when one or two syndesmotic screws are placed tricortically, the need for hardware removal is $10 \%$.

\section{Suture button fixation}

The suture button (marketed as the "TightRope" by Arthrex Inc, Naples, FL), a relatively new surgical implant, is a lowprofile system that consists of a No. 5 fiber-wire loop (Arthrex), which can be tensioned and secured between two metallic endobuttons placed against the outer cortices of the tibia and fibula (or fibular plate, if present). This device provides stabilization of the ankle mortise and reduces the need for subsequent procedures for device removal and, theoretically, late diastasis. A number of biomechanical studies have shown strength equivalent or only slightly inferior to screws [47, 68]. However, it is not clear whether the forces used in these studies approximate those transmitted during regular ambulation or that might occur during sporting activities.

While the clinical literature on suture button is encouraging $[44,51 \bullet \bullet, 52,69]$, it is also limited. In fact, a JAAOS review article on syndesmosis injuries published only 6 years before this article makes no mention of the suture button device [46]. The use of a suture device provides equivalent [69] or improved $[44,70]$ clinical outcomes, as compared with a fourcortical syndesmotic screw. Coetzee and Eberling [70] published their preliminary results of a prospective randomized trial comparing suture button with screw fixation. They suggest that "patients in the TightRope group have demonstrated better objective range-of-motion measurements and subjectively reported less stiffness and discomfort." AOFAS ankle/ hindfoot scores were higher in the suture button technique at an average of 18 months follow-up, although this did not reach statistical significance [70]. Naqvi et al. [51••] recently showed, in a prospective cohort study, that fixation with a suture button provides a more accurate method of syndesmotic stabilization, as compared with screw fixation, with equivalent clinical outcomes. Supporting evidence from previous studies [31, 63], syndesmotic malreduction was the most important independent predictor of clinical outcomes $[51 \bullet \cdot$. This underscores both the importance of accurate syndesmotic reduction and the potential benefit that suture button devices may play in optimizing reduction.

\section{Complications with TightRope}

Although an advantage of the suture button technique is mitigating the need for implant removal, there are several reports of infection, skin irritation, and granuloma formation warranting removal. In a recent study of 102 injuries treated with suture button fixation, $8 \%$ required removal for pain, infection, or implant loosening [53•]. Still, this is a lower removal rate than that associated with screw fixation [65]. Despite the higher than anticipated complication rate requiring removal of the suture button, it is done so through a small wound with few complications. Further well-designed, prospective studies are needed to confirm the long-term clinical outcomes of suture button fixation.

\section{Conclusions}

Traumatic injuries to the distal tibiofibular syndesmosis are relatively common and can be associated with ankle fractures or occur as purely ligamentous injuries, as often seen in contact sports. Syndesmotic injuries can create a diagnostic and therapeutic challenge for musculoskeletal physicians. Recent literature has added considerably to our understanding of injury mechanics and treatment outcomes with new technologies. Injury classification should facilitate prognosis, return 
to play and surgical decision making. The suture button device appears to have advantages over screws with improved reduction, maintenance of reduction, sufficient biomechanical strength, and no need for routine removal. However, further prospective and long-term clinical data on the suture button device are needed.

\section{Compliance with Ethics Guidelines}

Conflict of Interest Kenneth J. Hunt declares that he has no conflict of interest.

Human and Animal Rights and Informed Consent This article does not contain any studies with human or animal subjects performed by the author.

\section{References}

Papers of particular interest, published recently, have been highlighted as:

- Of importance

-. Of major importance

1. • Hermans JJ, Beumer A, de Jong TA, Kleinrensink GJ. Anatomy of the distal tibiofibular syndesmosis in adults: a pictorial essay with a multimodality approach. J Anat. 2010;217(6):633-45. Epub 2010/11/ 26. The article by Hermans et al. is a comprehensive pictorial description of syndesmosis anatomy as it relates to injury biomechanics.

2. Bartonicek J. Anatomy of the tibiofibular syndesmosis and its clinical relevance. Surgical Radiol Anat: SRA. 2003;25(5-6):379-86. Epub 2003/09/25.

3. Kim S, Huh YM, Song HT, Lee SA, Lee JW, Lee JE, et al. Chronic tibiofibular syndesmosis injury of ankle: evaluation with contrastenhanced fat-suppressed 3D fast spoiled gradient-recalled acquisition in the steady state MR imaging. Radiology. 2007;242(1):225-35. Epub 2006/12/23.

4. Takao M, Ochi M, Oae K, Naito K, Uchio Y. Diagnosis of a tear of the tibiofibular syndesmosis. J Bone Joint Surg Br. 2003;85(3):3249. Epub 2003/05/06.

5. McKeon KE, Wright RW, Johnson JE, McCormick JJ, Klein SE. Vascular anatomy of the tibiofibular syndesmosis. J Bone Joint Surg Am. 2012;94(10):931-8. Epub 2012/05/24.

6. Jelinek JA, Porter DA. Management of unstable ankle fractures and syndesmosis injuries in athletes. Foot Ankle Clin. 2009;14(2):27798. Epub 2009/06/09.

7. Fritschy D. An unusual ankle injury in top skiers. Am J Sports Med. 1989;17(2):282-5. discussion 5-6. Epub 1989/03/01.

8. Norkus S, Floyd R. The anatomy and mechanisms of syndesmotic ankle sprains. J Athl Train. 2001;36(1):68-73.

9. Pankovich AM. Maisonneuve fracture of the fibula. J Bone Joint Surg Am. 1976;58(3):337-42. Epub 1976/04/01.

10. Haraguchi N, Armiger R. A new interpretation of the mechanism of ankle fracture. J Bone Joint Surg Am. 2009;91:821-9.

11. Wei F, Villwock MR, Meyer EG, Powell JW, Haut RC. A biomechanical investigation of ankle injury under excessive external foot rotation in the human cadaver. J Biomech Eng. 2010;132(9):091001. Epub 2010/09/08.

12. Dattani R, Patnaik S, Kantak A, Srikanth B, Selvan TP. Injuries to the tibiofibular syndesmosis. J Bone Joint Surg Br. 2008;90(4):405-10. Epub 2008/04/02.
13. Femino JE, Vaseenon T, Phistkul P, Tochigi Y, Anderson DD, Amendola A. Varus external rotation stress test for radiographic detection of deep deltoid ligament disruption with and without syndesmotic disruption: a cadaveric study. Foot Ankle Int/ American Orthopaedic Foot and Ankle Society [and] Swiss Foot and Ankle Society. 2013;34(2):251-60. Epub 2013/02/16.

14. Wolfe MW, Uhl TL, Mattacola CG, McCluskey LC. Management of ankle sprains. Am Fam Physician. 2001;63(1):93-104. Epub 2001/ $02 / 24$.

15. Boytim MJ, Fischer DA, Neumann L. Syndesmotic ankle sprains. Am J Sports Med. 1991;19(3):294-8. Epub 1991/05/01.

16. Gerber JP, Williams GN, Scoville CR, Arciero RA, Taylor DC. Persistent disability associated with ankle sprains: a prospective examination of an athletic population. Foot Ankle Int/American Orthopaedic Foot and Ankle Society [and] Swiss Foot and Ankle Society. 1998;19(10):653-60. Epub 1998/11/04.

17. Teitz CC, Harrington RM. A biochemical analysis of the squeeze test for sprains of the syndesmotic ligaments of the ankle. Foot Ankle Int/ American Orthopaedic Foot and Ankle Society [and] Swiss Foot and Ankle Society. 1998;19(7):489-92. Epub 1998/08/07.

18. de Cesar PC, Avila EM, de Abreu MR. Comparison of magnetic resonance imaging to physical examination for syndesmotic injury after lateral ankle sprain. Foot Ankle Int/American Orthopaedic Foot and Ankle Society [and] Swiss Foot and Ankle Society. 2011;32(12):1110-4. Epub 2012/03/03.

19. Beumer A, van Hemert WL, Niesing R, Entius CA, Ginai AZ, Mulder PG, et al. Radiographic measurement of the distal tibiofibular syndesmosis has limited use. Clin Orthop Relat Res. 2004;423:22734. Epub 2004/07/03.

20. Harper MC, Keller TS. A radiographic evaluation of the tibiofibular syndesmosis. Foot Ankle. 1989;10(3):156-60. Epub 1989/12/01.

21. Pakarinen H, Flinkkila T, Ohtonen P, Hyvonen P, Lakovaara M, Leppilahti $\mathrm{J}$, et al. Intraoperative assessment of the stability of the distal tibiofibular joint in supination-external rotation injuries of the ankle: sensitivity, specificity, and reliability of two clinical tests. J Bone Joint Surg Am. 2011;93(22):2057-61. Epub 2012/01/21.

22. Shah AS, Kadakia AR, Tan GJ, Karadsheh MS, Wolter TD, Sabb B. Radiographic evaluation of the normal distal tibiofibular syndesmosis. Foot Ankle Int/American Orthopaedic Foot and Ankle Society [and] Swiss Foot and Ankle Society. 2012;33(10):870-6. Epub 2012/ $10 / 12$.

23. Summers HD, Sinclair MK, Stover MD. A reliable method for intraoperative evaluation of syndesmotic reduction. J Orthop Trauma. 2013;27(4):196-200. Epub 2013/03/27.

24. Oae K, Takao M, Naito K, Uchio Y, Kono T, Ishida J, et al. Injury of the tibiofibular syndesmosis: value of MR imaging for diagnosis. Radiology. 2003;227(1):155-61. Epub 2003/03/05.

25. Gardner M, Demetrakopoulos D, Briggs S, Helfet D, Lorich D. Malreduction of the tibiofibular syndesmosis in ankle fractures. Foot Ankle Int/American Orthopaedic Foot and Ankle Society [and] Swiss Foot and Ankle Society. 2006;27(10):788-92.

26. Hunt K, Githens M, Riley G, Kim M, Gold G. Foot and ankle injuries in sport imaging correlation with arthroscopic and surgical findings. Clin Sports Med. 2013;32:525-57.

27. Wolf BR, Amendola AA. Syndesmosis injuries in the athlete: when and how to operate. Curr Opin Orthop. 2002;31:151-4.

28. Scranton Jr PE. Sprains and soft tissue injuries. In: Pfefer G, editor. Chronic ankle pain in the athlete. Rosemont, Il: American Academy of Orthopaedic Surgeons; 2000. p. 3-20.

29. Beumer A, van Hemert WL, Swierstra BA, Jasper LE, Belkoff SM. A biomechanical evaluation of clinical stress tests for syndesmotic ankle instability. Foot Ankle In/American Orthopaedic Foot and Ankle Society [and] Swiss Foot and Ankle Society. 2003;24(4):358-63. Epub 2003/05/09.

30. Hermans JJ, Wentink N, Beumer A, Hop WC, Heijboer MP, Moonen $\mathrm{AF}$, et al. Correlation between radiological assessment of acute ankle 
fractures and syndesmotic injury on MRI. Skelet Radiol. 2012;41(7):787-801. Epub 2011/10/21.

31. Weening B, Bhandari M. Predictors of functional outcome following transsyndesmotic screw fixation of ankle fractures. J Orthop Trauma. 2005;19(2):102-8. Epub 2005/01/29.

32. Hunt KJ, George E, Harris AH, Dragoo JL. Epidemiology of syndesmosis injuries in intercollegiate football: incidence and risk factors from National Collegiate Athletic Association Injury Surveillance System Data from 2004-2005 to 2008-2009. Clin J Sport Med: Official Journal of the Canadian Academy of Sport Medicine. 2013. Epub 2013/01/24. This report of data from the NCAA injury surveillance system describes the incidence and epidemiology of ligamentous syndesmotic injuries in collegiate football players. Consistent with recent data, about a quarter of ankle sprains include syndesmotic injuries.

33. Kaplan LD, Jost PW, Honkamp N, Norwig J, West R, Bradley JP. Incidence and variance of foot and ankle injuries in elite college football players. Am J Orthop (Belle Mead NJ). 2011;40(1):40-4. Epub 2011/07/02.

34. Hopkinson WJ, St Pierre P, Ryan JB, Wheeler JH. Syndesmosis sprains of the ankle. Foot Ankle. 1990;10(6):325-30. Epub 1990/ $06 / 01$.

35. Waterman BR, Belmont Jr PJ, Cameron KL, Svoboda SJ, Alitz CJ, Owens BD. Risk factors for syndesmotic and medial ankle sprain: role of sex, sport, and level of competition. Am J Sports Med. 2011;39(5):992-8. Epub 2011/02/04.

36. Wright RW, Barile RJ, Surprenant DA, Matava MJ. Ankle syndesmosis sprains in national hockey league players. Am J Sports Med. 2004;32(8):1941-5. Epub 2004/12/02.

37. Nussbaum ED, Hosea TM, Sieler SD, Incremona BR, Kessler DE. Prospective evaluation of syndesmotic ankle sprains without diastasis. Am J Sports Med. 2001;29(1):31-5. Epub 2001/02/24.

38. Kennedy J. Surgical vs non-surgical treatment of syndesmotic injuries. J Orthop Trauma. 1990;14:232-40.

39. Taylor DC, Tenuta JJ, Uhorchak JM, Arciero RA. Aggressive surgical treatment and early return to sports in athletes with grade III syndesmosis sprains. Am J Sports Med. 2007;35(11):1833-8. Epub 2007/07/24.

40. Forsythe K, Freedman KB, Stover MD, Patwardhan AG. Comparison of a novel FiberWire-button construct versus metallic screw fixation in a syndesmotic injury model. Foot Ankle Int/American Orthopaedic Foot and Ankle Society [and] Swiss Foot and Ankle Society. 2008;29(1):49-54. Epub 2008/02/16.

41. Gardner R, Yousri T, Holmes F, Clark D, Pollintine P, Miles AW, et al. Stabilisation of the syndesmosis in the maisonneuve fracture - a biomechanical study comparing two-hole locking plate and quadricortical screw fixation. J Orthop Trauma. 2012. Epub 2012/05/12.

42. Hovis WD, Kaiser BW, Watson JT, Bucholz RW. Treatment of syndesmotic disruptions of the ankle with bioabsorbable screw fixation. J Bone Joint Surg Am. 2002;84-A(1):26-31. Epub 2002/01/17.

43. Thordarson DB, Samuelson M, Shepherd LE, Merkle PF, Lee J. Bioabsorbable versus stainless steel screw fixation of the syndesmosis in pronation-lateral rotation ankle fractures: a prospective randomized trial. Foot Ankle Int/American Orthopaedic Foot and Ankle Society [and] Swiss Foot and Ankle Society. 2001;22(4):335-8. Epub 2001/05/17.

44. Thornes B, Shannon F, Guiney AM, Hession P, Masterson E. Suture-button syndesmosis fixation: accelerated rehabilitation and improved outcomes. Clin Orthop Relat Res. 2005;431:207-12. Epub 2005/02/03

45. Xenos JS, Hopkinson WJ, Mulligan ME, Olson EJ, Popovic NA. The tibiofibular syndesmosis. Evaluation of the ligamentous structures, methods of fixation, and radiographic assessment. J Bone Joint Surg Am. 1995;77(6):847-56. Epub 1995/06/01.

46. Zalavras C, Thordarson D. Ankle syndesmotic injury. J Am Acad Orthop Surg. 2007;15(6):330-9. Epub 2007/06/06.
47. Soin SP, Knight TA, Dinah AF, Mears SC, Swierstra BA, Belkoff $\mathrm{SM}$. Suture-button versus screw fixation in a syndesmosis rupture model: a biomechanical comparison. Foot Ankle Int/American Orthopaedic Foot and Ankle Society [and] Swiss Foot and Ankle Society. 2009;30(4):346-52. Epub 2009/04/10.

48. Wikeroy AK, Hoiness PR, Andreassen GS, Hellund JC, Madsen JE. No difference in functional and radiographic results 8.4 years after quadricortical compared with tricortical syndesmosis fixation in ankle fractures. J Orthop Trauma. 2010;24(1):17-23.

49. Degroot H, Al-Omari AA, El Ghazaly SA. Outcomes of suture button repair of the distal tibiofibular syndesmosis. Foot Ankle Int/ American Orthopaedic Foot and Ankle Society [and] Swiss Foot and Ankle Society. 2011;32(3):250-6. Epub 2011/04/12.

50. Teramoto A, Suzuki D, Kamiya T, Chikenji T, Watanabe K, Yamashita T. Comparison of different fixation methods of the suture-button implant for tibiofibular syndesmosis injuries. Am J Sports Med. 2011;39(10):2226-32. Epub 2011/07/20.

51. •• Naqvi GA, Cunningham P, Lynch B, Galvin R, Awan N. Fixation of ankle syndesmotic injuries: comparison of TightRope fixation and syndesmotic screw fixation for accuracy of syndesmotic reduction. Am J Sports Med. 2012;40(12):2828-35. Epub 2012/10/12. Welldesigned, prospective cohort study indicating that fixation with TightRope provides a more accurate method of syndesmotic stabilization, as compared with syndesmotic screws. Consistent with previous data, syndesmotic malreduction is the most important independent predictor of clinical outcomes.

52. Naqvi GA, Shafqat A, Awan N. TightRope fixation of ankle syndesmosis injuries: clinical outcome, complications and technique modification. Injury. 2012;43(6):838-42. Epub 2011/11/01.

53. • Storey P, Gadd RJ, Blundell C. Complications of suture button ankle syndesmosis stabilization with modifications of surgical technique. Foot Ankle Int/American Orthopaedic Foot and Ankle Society [and] Swiss Foot and Ankle Society. 2012;33(9):717-21. Epub 2012/09/22. This retrospective case series of 102 patients describes complications associated with the TightRope method to reduce their incidence.

54. Markolf KL, Jackson SR, McAllister DR. Syndesmosis fixation using dual $3.5 \mathrm{~mm}$ and $4.5 \mathrm{~mm}$ screws with tricortical and quadricortical purchase: a biomechanical study. Foot Ankle Int/ American Orthopaedic Foot and Ankle Society [and] Swiss Foot and Ankle Society. 2013. Epub 2013/02/14.

55. • Egol KA, Pahk B, Walsh M, Tejwani NC, Davidovitch RI, Koval KJ. Outcome after unstable ankle fracture: effect of syndesmotic stabilization. J Orthop Trauma. 2010;24(1):7-11. Epub 2009/12/26. This retrospective review of 347 patients showed that those who required syndesmotic stabilization in addition to fracture fixation had poorer outcomes at 12 months, as compared with patients who required malleolar fracture fixation alone.

56. • Symeonidis P, Iselin L, Chehade M, Stavrou P. Common Pitfalls in Syndesmotic Rupture Management : A Clinical Audit. Foot Ankle Int/American Orthopaedic Foot and Ankle Society [and] Swiss Foot and Ankle Society. 2013;34(originally published online 18 January 2013):345. This retrospective comparative series conducted at a level 1 trauma center suggests that the reoperation rate for syndesmotic fixation may be higher than previously thought. The authors emphasize the three important points in the management of these injuries: suspect the injury, document the stability of the syndesmosis, and reduce the fibula anatomically.

57. Beumer A, Campo MM, Niesing R, Day J, Kleinrensink GJ, Swierstra BA. Screw fixation of the syndesmosis: a cadaver model comparing stainless steel and titanium screws and three and four cortical fixation. Injury. 2005;36(1):60-4. Epub 2004/12/14.

58. Hansen M, Le L, Wertheimer S, Meyer E, Haut R. Syndesmosis fixation: analysis of shear stress via axial load on $3.5-\mathrm{mm}$ and $4.5-$ $\mathrm{mm}$ quadricortical syndesmotic screws. J Foot Ankle Surg: Official Publication of the American College of Foot and Ankle Surgeons. 2006;45(2):65-9. Epub 2006/03/04. 
59. Thompson MC, Gesink DS. Biomechanical comparison of syndesmosis fixation with 3.5- and 4.5-millimeter stainless steel screws. Foot Ankle Int/American Orthopaedic Foot and Ankle Society [and] Swiss Foot and Ankle Society. 2000;21(9):736-41. Epub 2000/10/07.

60. Nousiainen MT, McConnell AJ, Zdero R, McKee MD, Bhandari M, Schemitsch EH. The influence of the number of cortices of screw purchase and ankle position in Weber $\mathrm{C}$ ankle fracture fixation. $\mathrm{J}$ Orthop Trauma. 2008;22(7):473-8. Epub 2008/08/02.

61. Bragonzoni L, Russo A, Girolami M, Albisinni U, Visani A, Mazzotti $\mathrm{N}$, et al. The distal tibiofibular syndesmosis during passive foot flexion. RSA-based study on intact, ligament injured and screw fixed cadaver specimens. Arch Orthop Trauma Surg. 2006;126(5):304-8. Epub 2006/03/29.

62. Tornetta 3rd P, Spoo JE, Reynolds FA, Lee C. Overtightening of the ankle syndesmosis: is it really possible? J Bone Joint Surg Am. 2001;83-A(4):489-92. Epub 2001/04/24.

63. Sagi HC, Shah AR, Sanders RW. The functional consequence of syndesmotic joint malreduction at a minimum 2-year follow-up. J Orthop Trauma. 2012;26(7):439-43. Epub 2012/02/24.

64. Schepers T. To retain or remove the syndesmotic screw: a review of literature. Arch Orthop Trauma Surg. 2011;131(7):879-83. Epub 2010/ 12/17. This literature review describes recent data on the role of hardware removal for syndesmotic screws. The current literature suggests that hardware removal should be reserved for intact screws that cause hardware irritation or reduced range of motion after 4-6 months.

65. Hamid N, Loeffler BJ, Braddy W, Kellam JF, Cohen BE, Bosse MJ. Outcome after fixation of ankle fractures with an injury to the syndesmosis: the effect of the syndesmosis screw. J Bone Joint Surg Br. 2009;91(8):1069-73. Epub 2009/08/05.

66. Manjoo A, Sanders DW, Tieszer C, MacLeod MD. Functional and radiographic results of patients with syndesmotic screw fixation: implications for screw removal. J Orthop Trauma. 2010;24(1):2-6. Epub 2009/12/26.

67. Schepers T, Van Lieshout EM, de Vries MR, Van der Elst M. Complications of syndesmotic screw removal. Foot Ankle Int/ American Orthopaedic Foot and Ankle Society [and] Swiss Foot and Ankle Society. 2011;32(11):1040-4. Epub 2012/02/22.

68. Thornes B, Walsh A, Hislop M, Murray P, O'Brien M. Sutureendobutton fixation of ankle tibio-fibular diastasis: a cadaver study. Foot Ankle Int/American Orthopaedic Foot and Ankle Society [and] Swiss Foot and Ankle Society. 2003;24(2):142-6. Epub 2003/03/12.

69. Cottom JM, Hyer CF, Philbin TM, Berlet GC. Transosseous fixation of the distal tibiofibular syndesmosis: comparison of an interosseous suture and endobutton to traditional screw fixation in 50 cases. J Foot Ankle Surg: Official Publication of the American College of Foot and Ankle Surgeons. 2009;48(6):620-30. Epub 2009/10/28.

70. Coetzee J, Eberling P. Treatment of syndesmosis disruptions with TightRope fixation. Tech Foot Ankle Surg. 2008;7:196-201.

71. Pneumaticos SG, Noble PC, Chatziioannou SN, Trevino SG. The effects of rotation on radiographic evaluation of the tibiofibular syndesmosis. Foot Ankle Int/American Orthopaedic Foot and Ankle Society [and] Swiss Foot and Ankle Society. 2002;23(2):107-11. Epub 2002/02/23. 